\title{
A Study of Iris Segmentation Methods using Fuzzy C- Means and K-Means Clustering Algorithm
}

\author{
S.Jayalakshmi ${ }^{1}$, M.Sundaresan ${ }^{2}$ \\ ${ }^{1}$ Research Scholar, Department of Information Technology, Bharathiar University, Coimbatore \\ ${ }^{2}$ Associate professor and Head, Department of Information Technology, Bharathiar University, Coimbatore
}

\begin{abstract}
A biometric system provides automatic identification of an individual based on a unique feature or characteristic possessed by the individual. Among most of the biometric methods, Iris recognition is regarded as the most reliable and accurate biometric identification system. The performance of iris recognition system highly depends on the accurate segmentation. For the Iris Segmentation there is a lot of methods that have been proposed in several decades. This present research work explores the Iris Segmentation process along with Fuzzy C-Means algorithm and K-Means clustering algorithm. The segmentation technique presented in this paper includes image acquisition, filtering, inner boundary localization, outer boundary localization and exclusion of eyelids and eyelashes. In this paper segmentation process are implemented using images from CASIA iris dataset image available on net. All the algorithms are implemented separately and the results are obtained. As a result Segmentation using FCM produces high accuracy rate of $98.20 \%$ and low error rate when compared to methods.
\end{abstract}

\section{Index Terms}

Iris Recognition, Segmentation, Boundary localization, Fuzzy C-Means clustering algorithm, K-Means clustering algorithm

\section{INTRODUCTION}

Image processing is a rapidly growing area of computer science. Its growth has been achieved by the technological advances in digital imaging, computer processors and mass storage devices. Fields which have traditionally used analog imaging are now switching to digital systems, as it provides more flexibility and affordability.

One of the most developing areas in modern world is Biometrics, which was developed for replacing the traditional identification systems. Biometric system works by first capturing a sample of the feature such as recording a digital sound signal for voice recognition or taking a digital color image for face or iris recognition. The sample is then transformed using some sort of mathematical function into a biometric template. The biometric template will provide a normalized efficient and highly discriminating representation of the feature, which can then be objectively compared with other templates in order to determine identity.

A biometric system provides automatic recognition of an individual based on some sort of unique features or characteristic possessed by the individual such as fingerprints, facial features, voice, hand geometry, handwriting, the retina and the one presented in this paper, the iris.

Among many identification patterns, iris recognition plays an important role and it has drawn considerable interest. In last two decades researchers are interested in working with biometrics especially iris recognition which draws more interest and attention for the person identification, because of its potential applications in the area such as National border controls [1], where the iris is used as the living passport, Computer login, where the iris acts as a living password, Cellphone and other wireless device based on authentication, Secure access to bank accounts at cash machines, Ticketless travel where it acts as an authentication of rights to services, secure financial transaction, etc.,

Image segmentation is one of the most important steps leading to the analysis of processed image data, which refers to grouping of similar pixels together and separating the particular portion of the image for the purpose of identification. Its main goal is to divide an image into parts that have strong correlation with objects or areas of the real world contained in the image.

The main aim of segmentation is to simplify and/or change the representation of an image into something that is more meaningful and easier to analyze and is to cluster pixels into salient image regions [2], i.e., regions corresponding to individual surfaces, objects, or natural parts of objects.

Iris Segmentation plays a major role in the process of Iris recognition. The accurate recognition/classification is mainly based on proper segmentation of Iris and Pupil. The main purpose of segmentation is to localize the two iris boundaries namely, inner boundary of iris-pupil and outer iris-sclera and to localize eyelids.

The process of segmentation may be inaccurate due to the factors which affects the quality of eye images. Poor quality images which cause failure in the segmentation of iris results failure in recognition.

\section{METHODOLOGY}

Nowadays people need to prove their identity for security purpose. Among many identification system, Iris recognition is widely used as it is more fast and accurate than other classification process. Each individual possess unique pattern of iris and this feature helps to identify a person quickly when compared to other features [3].

The main objective of this research work is to measure the performance of Fuzzy C-Means and K-Means clustering algorithms and to find which gives better performance and accuracy in Iris Segmentation.

In the existing work, segmentation of iris has been done using Hough transform. Circular Hough Transform which is used for finding/localizing the inner and outer boundary of iris. Linear Hough Transform is used for removing the upper and lower eyelids, in the case of occluded eye images [4].

In the present work, along with the general Iris Segmentation process several algorithms are added subsequently and their performance are measured. It will enable us to find the algorithm which produces the better results. The following are the steps of proposed Iris Segmentation.

\subsection{Image Extraction}

This is the first step in the process of Iris Segmentation. The eye images can be obtained in real time by capturing the 
images in digital camera or through web camera. For this study the images are collected from the CASIA dataset. There are several versions of dataset that are available in CASIA dataset.

\subsection{Pre-Processing}

The success of segmentation depends on the imaging quality of eye images. Images in the CASIA iris database do not contain specular reflections due to the use of near infra-red light for illumination [5]. In order to overcome this type of image quality factors, the image should be pre-processed to make the segmentation process more accurate.

\subsection{Finding the Center}

The center of the image is found in the next stage, which helps to calculate the center of the image by obtaining the center of each row and column. It is found using an integrodifferential operator.

\subsection{Finding the Inner and Outer Boundary}

The next step in the process is localizing the Inner [Pupil] and outer [Sclera] boundary of the eye image. It is found by using Circular Hough Transform.

\subsection{Iris Segmentation process}

Two algorithms are implemented in proposed research work so as to increase the accuracy of Iris Segmentation.

\section{K-Means Clustering Algorithm}

The MacQueen's k-means algorithm [6] is a popular clustering algorithm in which the number of clusters $(\mathrm{k})$ is known. It is an iterative process that assigns patterns to the closest cluster using a distance function (such as the Euclidean distance measure). The basic algorithm is described below.

1. Define the number of clusters $k$.

2. Initialize (randomly) the clusters prototypes

$$
\mathbf{p}_{\mathrm{i}}(\mathrm{i}=1 \ldots . . \mathrm{k}) \text {. }
$$

3. For each pattern $\mathbf{x}$, assign $\mathbf{x}$ to the nearest cluster

$$
\mathbf{p}_{\mathrm{i}}(\mathrm{i}=1 \ldots \mathrm{k}) \text {. }
$$

4. Re compute

$$
\text { pi }(i=1 \ldots . . . k) \text {. }
$$

5. Repeat steps 3 and 4 until the prototypes do not change.

The algorithm used is similar to the one described above but uses a function to derive weights for the distance function. This algorithm is implemented and the results are obtained.

\section{Fuzzy C-Means algorithm}

Fuzzy c-means (FCM) algorithm is the most popular method used in image segmentation because it has robust characteristics for ambiguity [7] and can retain much more information than hard segmentation methods. Although the conventional FCM algorithm works well on most noise-free images, it has a serious limitation, i.e., it does not incorporate any information about spatial context, which cause it to be sensitive to noise and imaging artifacts. This algorithm is an iterative clustering method that produces an optimal $\mathrm{c}$ partition by minimizing the weighted within group sum of squared error objective function JFCM.

$$
\mathrm{JFCM}_{=} \sum_{k=1}^{n} \sum_{i=1}^{c}\left(\mathrm{u}_{\mathrm{ik}}\right)^{\mathrm{q}} \mathrm{d}^{2}\left(\mathrm{x}_{\mathrm{k}}, \mathrm{v}_{\mathrm{i}}\right)
$$

where $X=\{x 1, x 2, \ldots, x n\} \leq R p$ is the data set in the $p$ dimensional vector space, is the number of data items, $\mathrm{c}$ is the number of clusters with $2 \leq \mathrm{c}<\mathrm{n}$, uik is the degree of membership of $\mathrm{xk}$ in the ith cluster, $\mathrm{q}$ is the weighting exponent on each fuzzy membership, vi is the prototype of the centre of cluster $\mathrm{i}, \mathrm{d} 2(\mathrm{xk}, \mathrm{vi})$ is a distance measure between object xk and cluster centre vi. A solution of the object function JFCM can be obtained via an iterative process, which is carried as follows:

- $\quad$ set values for $c, q$, and $\mathrm{e}$,

- $\quad$ initialize the fuzzy partition matrix ,

- $\quad$ set the loop counter $b=0$,

- $\quad$ calculate the $c$ cluster centers $\left\{v_{i}^{(b)}\right\}$ with $\mathrm{U}^{(\mathrm{b})}$

$$
v_{i}^{(b)}=\frac{\sum_{k=1}^{n}\left(u_{i k}^{(b)}\right)^{q} x_{k}}{\sum_{k=1}^{n}\left(u_{i k}^{(b)}\right)^{q}}
$$

- calculate the membership $\mathrm{U}^{(\mathrm{b}+1)}$, For $k=1$ to $n$, calculate the following:, $I_{k}=\left\{i \mid 1<=i<=c ; d_{i k}=\| x_{k}-\right.$ $\left.\mathrm{v}_{\mathrm{i}} \|=0\right\}, \sim \mathrm{I}_{\mathrm{k}}=\{1,2, \ldots \ldots \mathrm{c}\}-\mathrm{I}_{\mathrm{k},}$, for the $k$ th column of the matrix, compute new membership values, and if $\mathrm{I}_{\mathrm{k}}=\emptyset$, then

$$
u_{i k}^{(b+1)}=\frac{1}{\sum_{j=1}^{c}\left(\frac{d_{i j}}{d_{j k}}\right)^{\frac{2}{q-1}}}
$$

else $u_{i k}^{(b+1)}=0$ for all $\mathrm{i} \epsilon \sim \mathrm{I}_{\mathrm{k}}$ and $\sum_{i \in I_{K}} u_{i k}^{(b+1)}=1$, next k,

- $\quad$ if $\left\|\mathrm{U}^{\mathrm{b}}-\mathrm{U}^{(\mathrm{b}+1)}\right\|<\varepsilon$, stop; otherwise set $\mathrm{b}=\mathrm{b}+1$ and go to step 4.

This algorithm [8] is then proposed by minimizing this new objective function according to the zero gradient condition, which can handle both the feature space information and spatial information during segmentation.

As a result the segmented iris image is obtained by using these algorithms and the results are obtained.

\section{IMPLEMENTATION AND RESULTS}

The first stage of iris recognition is to isolate the actual iris region in a digital eye image. The iris region can be approximated by two circles, one for the iris/sclera boundary and another, interior to the first, for the iris/pupil boundary [9]. The eyelids and eyelashes normally occlude the upper and lower parts of the iris region [10]. Also, specular reflections can occur within the iris region corrupting the iris pattern. A technique is required to isolate and exclude these artifacts as well as locating the circular iris region.

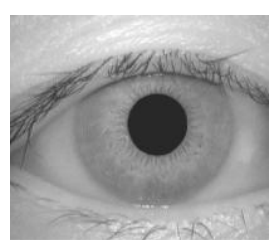

(a)

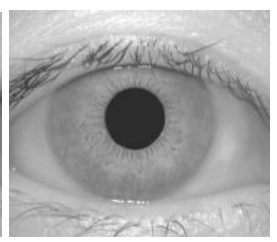

(b)
Fig 1: Images obtained from CASIA dataset

The success of segmentation depends on the imaging quality of eye images. Images in the CASIA iris database, Figure 1, do not contain specular reflections due to the use of near 
infra-red light for illumination. For that the pre-processing stage is involved in the segmentation process in order to improve the quality of the image obtained.

After the pre-processing stage, the center of the iris image is to be found as shown in Figure 2.

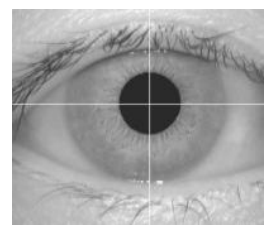

Fig 2: Image after the center is found

It enables to easily identify the inner and outer boundary which is to be identified next in the pre-processing stage.

The inner boundary and outer boundary of the iris is found as shown in Figure 3, using circle-hough transform [11]. Using this methodology the inner pupil boundary was found and also the outer sclera boundary was located.

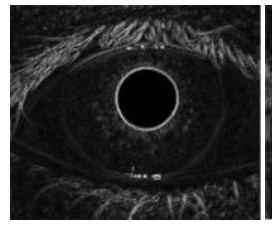

(a)

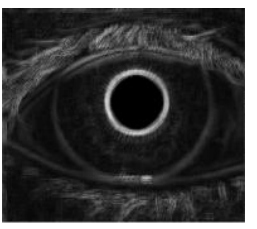

(b)
Fig 3: (a) Image obtained after finding the inner boundary (Pupil); (b) Image obtained after finding the outer boundary of iris (Sclera)

The threshold value [12] is calculated in order to find whether the eyelids and eyelashes are occluded in the iris. If the maximum hough space is less than the set threshold value then there is no occlusion occurred and if the maximum hough space is higher than the set threshold value, then it is considered as the occlusion is occurred.

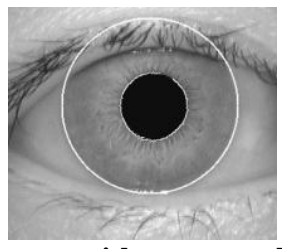

Fig 4: Original image with segmented boundary of Iris

After all this pre-processing methods [13], a new approach of this research work is implemented. FCM and K-Means algorithm is used for segmenting the iris from the eye image. The two algorithms are implemented separately and the results are observed. This makes us to identify the algorithm which produces accurate results.

Finally the segmented iris image is obtained in both the cases. Among that segmentation using Fuzzy C-Means algorithm produced better results, when compared to K-Means clustering algorithms.

Segmentation of Iris using FCM gave $98.20 \%$ of accuracy with low error rate within a considerable amount of time and K-Means clustering algorithm [14] took time to calculate but produced $84.98 \%$ accuracy rate. After segmentation, the segmented iris image is located on the original iris image obtained as shown in Figure 4.

\section{RESULTS AND DISCUSSIONS}

Nowadays, iris recognition becomes a major research area for the researchers as it is more accurate in identifying the individuals. Accurate identification is based on proper segmentation of iris. Several methods are proposed for segmentation in several decades. In past, researchers used Circle Hough Transform [15] for localizing the inner (irispupil) and outer boundary (iris-sclera) of iris and linear hough transform for removing the upper and lower eyelids and eyelashes occluded on the iris. It results in the percentage accuracy of $97.5 \%$ in segmentation of iris.

In the proposed work, two different algorithms are used separately and their performances are measured. On comparing the results of both algorithms, segmentation using Fuzzy C-Means algorithm produces low error rate and high percentage of accuracy when compared to the methods using K-Means clustering. FCM algorithm produces $98.2 \%$ of accuracy within a considerable amount of time.

For determining the performance of these two algorithms, Root Mean Square Error [16], Mean Absolute Error [17], Time taken for execution and Percentage of accuracy are calculated. The Root Mean Square Error and Mean Absolute Error are calculated using the following formula.

$$
\begin{gathered}
\mathrm{RMSE}=\sqrt{\frac{\sum_{t=1}^{n}\left(x_{1}, t-x_{2}, t\right)^{2}}{n}} \\
\mathrm{MAE}=\frac{1}{n} \sum_{i=1}^{n}\left|f_{i}-y_{i}\right|=\frac{1}{n} \sum_{i=1}^{n}\left|e_{i}\right|
\end{gathered}
$$

For the implementation of this proposed work, five images are taken which are collected from CASIA iris image dataset. The values of RMSE and MAE which obtained are shown in Table 1 and Table 2 respectively.

Table 1: RMSE values

\begin{tabular}{|l|c|c|}
\hline & K-MEANS & FUZZY C-MEANS \\
\hline IMAGE1 & 16.51 & 1.17 \\
\hline IMAGE2 & 17.03 & 1.75 \\
\hline IMAGE3 & 16.92 & 2.01 \\
\hline IMAGE4 & 16.34 & 1.58 \\
\hline IMAGE5 & 16.28 & 1.99 \\
\hline
\end{tabular}

From the values, we can easily identify that FCM algorithm produces low Root Mean Square Error values when compared to K-Means algorithm.

The Mean Absolute Error values also been calculated and tabulated as follows:

Table 2: Mean Absolute Error values

\begin{tabular}{|l|c|c|}
\hline & K-MEANS & FUZZY C-MEANS \\
\hline IMAGE1 & 199 & 92 \\
\hline IMAGE2 & 193 & 80 \\
\hline IMAGE3 & 206 & 87 \\
\hline IMAGE4 & 218 & 69 \\
\hline IMAGE5 & 202 & 84 \\
\hline
\end{tabular}


By calculating the values it is clear that Fuzzy C-Means algorithm produces low MAE when compared to K-Means clustering algorithm.

Table 3: Percentage of Accuracy

\begin{tabular}{|l|c|c|}
\hline & K-MEANS & FUZZY C-MEANS \\
\hline IMAGE1 & $84.82 \%$ & $98.20 \%$ \\
\hline IMAGE2 & $83.95 \%$ & $97.89 \%$ \\
\hline IMAGE3 & $85.79 \%$ & $98.05 \%$ \\
\hline IMAGE4 & $84.02 \%$ & $98.32 \%$ \\
\hline IMAGE5 & $83.54 \%$ & $97.06 \%$ \\
\hline
\end{tabular}

The percentage of accuracy for the algorithm is calculated based on the error rate to determine the better algorithm for segmentation. Table 3 shows the percentage of accuracy on using both Fuzzy C-Means algorithm and K-Means clustering algorithm.

From the table given we can observe that FCM produces $98.20 \%$ of accuracy while K-Means algorithm produces $83.95 \%$, comparatively which is lesser than the other.

Table 4: Time taken in Seconds

\begin{tabular}{|l|c|c|}
\hline & K-MEANS & FUZZY C-MEANS \\
\hline IMAGE1 & 12 & 35 \\
\hline IMAGE2 & 14 & 39 \\
\hline IMAGE3 & 13 & 45 \\
\hline IMAGE4 & 12 & 41 \\
\hline IMAGE5 & 11 & 49 \\
\hline
\end{tabular}

The time for segmentation of iris from the image is obtained. The time is calculated in seconds, from the initially obtaining the image and till segmenting the iris from the image. Table 4 shows the values of time in seconds for the proposed method of segmentation.

Though it takes some more time more than other algorithm it gives better accuracy rate within a considerable amount of time.

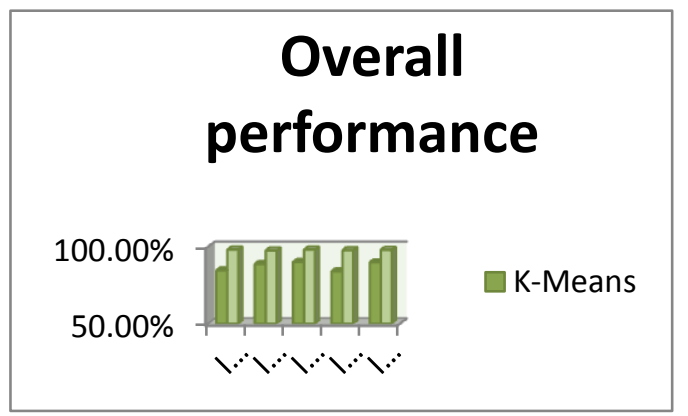

Fig 5: Overall performance

By observing the overall performance, the proposed method of segmentation using FCM produces better results when compared to K-Means clustering algorithm which is depicted in the Figure 5. It produces high accuracy rate of $98.20 \%$ and lowest error rate of 1.85 . Thus, from the analysis of the results in the proposed work, it is clear that the Fuzzy C-Means algorithm produces better result for the process of Iris Segmentation.

\section{CONCLUSION}

In this research work, the algorithms used for segmentation of iris from the image obtained are implemented using powerful tool, MATLAB. For pre-processing canny edge detection method is used along with high pass filters. Then circle hough transform is used to localize the inner and outer boundary of iris. Then two algorithms Fuzzy C-Means algorithm and KMeans algorithm are implemented separately along with normal segmentation method using the powerful tool, MATLAB. The performances are observed and the results are obtained. Among them Fuzzy C-Means algorithm produces low error rate when compared to others. It produces high percentage of accuracy $98.20 \%$ and low error rate of 1.80 when compared to other algorithm within a considerable amount of time.

\section{REFERENCES}

[1] Rafael C. Gonzalez and Richard E Woods (2009), "Digital image processing", ISBN 978-81-317-2695-2, Pearson Education.

[2] Jayaraman, Essakkirajan and Veerakumaran.(2009), "Digital image processing", ISBN(13):978-0-07-0144789, ISBN(10): 0-07-014479-6, Tata McGraw Hill Education Private limited.

[3] E. Wolff,"Anatomy of the Eye and Orbit",7 edition. H. K. Lewis \& Co. LTD, 1976.

[4] R. Wildes, "OIris recognition: an emerging biometric technology", Proceedings of the IEEE, Vol. 85, No. 9, 1997.

[5] J. Daugman, "Biometric personal identification system based on iris analysis", United ETRI Journal, Vol. 23, No. 2, Korea, 2001. States Patent, Patent Number: 5,291,560, 1994.

[6] J. Daugman, "High confidence visual recognition of persons by a test of statistical independence",IEEE Transactions on Pattern Analysis and Machine Intelligence, Vol. 15, No. 11, pp. 2-3,1993.

[7] Jianbo Shi and Jitendra Malik, "Normalized Cuts and Image Segmentation", IEEE transactions on pattern analysis and machine intelligence, vol. 22, no. 8, August 2000.

[8] Jing Huang, XingeYou, YuanYanTang, LiangDu, YuanYuan, "A novel iris segmentation using radialsuppression edge detection", journal Signal Processing 89 (2009) 2630-2643, Pp. 12-35.

[9] Amjad Zaim, "Automatic segmentation of iris images for the purpose of Identification", 0-7803-9134-9/05/\$20.00 (C)2005 IEEE.

[10] H. Proenca and L.A. Alexandre, "Iris segmentation methodology for non-cooperative recognition", Published in IEE proceedings vision, Image \& Signal Processing, April, 2006, Volume 153, Issue 2, Pp.99205.

[11] Xiaofei Hu, V. Paul Pauca, and Robert Plemmons, "Iterative Directional Ray-based Iris Segmentation for Challenging Periocular Images", 0-7803-86221/04/\$20.00 @2004 IEEE, Pp. 35-67. 
[12] Peihua Li, Xiaomin Liu, Lijuan Xiao and Qi Song, "Robust and Accurate Iris Segmentation in Very Noisy Iris Images", Preprint submitted to Image and Vision Computing April 26, 2009.

[13] Nicolaie Popescu-Bodorin, "Circular Fuzzy Iris Segmentation", Image and Vision Computing 28 (2009) 278-284.

[14] Dr. K Revathy, "Applying EM Algorithm for Segmentation of Textured Images", Proceedings of the World Congress on Engineering 2007 Vol I, WCE 2007, July 2 - 4, 2007, London, U.K.
[15] Mahmoud Mahlouji, Ali Noruzi, "Human Iris Segmentation for Iris Recognition in Unconstrained Environments", IJCSI International Journal of Computer Science Issues, Vol. 9, Issue 1, No 3, January 2012.

[16] Przemysław Strzelczyk, "Robust and Accurate Iris Segmentation Algorithm for Color and Noisy Eye Images “, Journal of Telecommunications and Information Technology, 4/2010.

[17] Mahmoud Mahlouji, Ali Noruzi, "Human Iris Segmentation for Iris Recognition in Unconstrained Environments", IJCSI International Journal of Computer Science Issues, Vol. 9, Issue 1, No 3, January 2012. 